\title{
Evaluation of a novel malleable, biodegradable osteoconductive composite in a rabbit cranial defect model
}

\author{
Tim-Mo Chen ${ }^{a}$, Chun-Hsu Yao ${ }^{b}$, Hsian-Jenn Wang ${ }^{a}$, Giuen-Hsueng Chou ${ }^{a}$, Tze-Wen Lee ${ }^{a}$, \\ Feng-Huei Lin ${ }^{b, *}$ \\ a Bum Center, Department of Surgern, Tri-Senice General Hospital, National Defense Medical Center, Taipei, Tainan \\ 'Center for Biomedical Engineering, College of Medicine, National Taiwan University, Taipei, Taiwan \\ Received 30 October 1997; received in revised form 22 December 1997; accepted 12 January 1998
}

\begin{abstract}
The ceramic form of calcium phosphate osteoconductive material such as hydroxyapatite is brittle, non-malleable and non-degradable, and these mechanical properties limit its clinical application in calvarium reconstruction. To improve these properties, we developed a malleable, biodegradable osteoconductive composite composed of tricalcium phosphate particles bound by a gelatin which is set by glutaraldehyde mediated cross-linking. The composite was implanted into a $15 \times 15 \mathrm{~mm}$ full-thickness, calvarial defect in 20 rabbits for up to 3 months. Twelve rabbits were left unreconstructed as controls. Specimens were retrieved at 2 weeks, 1, 2 and 3 months. Five reconstructed and 3 unreconstructed rabbits were examined for each time period. The assessment included a series of post operative gross examinations, radiographs and histologic evaluations. We are able to demonstrate that this composite is (1) biocompatible, with little tissue reaction; (2) osteoconductive, with progressive growth of new bone into the calvarial defect; (3) biodegradable, with progressive replacement of the composite by new bone, acellular matrix and bone-like material. Replacement of this composite by new bone is postulated to occur by a combination of osteoconduction and biodegradation. These results indicate that further experimental research to combine this malleable, biodegradable, osteoconductive composite with an osteoinductive agent such as bone morphogenetic protein may generate new biomaterial for full-thickness calvarial defect reconstruction. (c) 1998 Elsevier Science S.A. All rights reserved.
\end{abstract}

Kevwords: Biodegradable composite; Osteoconductive composite; Rabbit cranial defect model

\section{Introduction}

The head is not infrequently a contact of entry site in high tension electric bums. With loss of scalp and involvement of the underlying calvarium, the modern approach is early excision of the necrotic scalp and well-vascularized flap coverage without regard for the degree of bone devitalization [1]. This concept regards devitalized calvarium as salvageable, acting as an in-situ bone graft. However, this approach is not always successful. Sometimes, the devitalized calvarium may become heavily colonized and osteomyelitic. In this situation, excision of the full-thickness calvarium is mandatory.

After excision of the full-thickness calvarium, reconstruction of the calvarial bone is indicated to provide brain protection and aesthetic contour. The two most widely used materials for calvarial bone defect reconstruction are autogenous bone graft and methyl methacrylate. Each is efficacious

\footnotetext{
* Corresponding author. Tel.: + 886-2-3970800 ext 1449; Fax: + 886-23940049; E-mail: double@ha.me.ntu.edu.tw
}

in full-thickness calvarial bone defect repair, but both are less than ideal. Autogenous bone grafts can undergo unpredictable resorption, are of limited quantity and may result in secondary donor site morbidity [2-5]. Methyl methacrylate can cause significant inflammatory response and fibrous encapsulation of the implant, resulting in possible infection, loosening, and exposure of the implant [6]. Allogenic bank bone from human cadavers is another alternative to the autograft [7], however, possible rejection and potential pathogens transmission such as HIV and hepatitis viruses diminish the clinical utility. Thus, the search for synthetic bone-promoting biomaterials as alternatives to attograft and allograft remains an important topic in medical research.

In the fields of medicine and biomedical engineering, much effort has been directed to the development of osteoconductive materials composed of various calcium phosphate compounds because of close chemical and crystal resemblance of these materials to natural bone mincral [8-15]. The osteoconductive materials would aid in the healing of the bone defect by acting, preferably temporarily, as a scattold for 
bone ingrowth. The mostly widely investigated calcium phosphate compounds are tricalcium phosphate $\mathrm{Ca}_{3}\left(\mathrm{PO}_{4}\right)_{2}$ and hydroxyapatite $\mathrm{Ca}_{30}\left(\mathrm{PO}_{4}\right)_{6}(\mathrm{OH})_{2}$. They differ not only in their composition but also in their rate of resorption $[16,17]$. Tricalcium phosphate degrades twice as fast as hydroxyapatite. A biodegradable osteoconductive matrix will not produce a permanent indwelling foreign body and will eventually provide greater room for bone ingrowth. When shaped in porous blocks, calcium phosphate compounds are brittle, with average strengths comparable to or less than that of cancellous bone [18], and can be easily fractured. In addition, these blocks are inherently non-malleable and difficult to shape so that they accurately replace missing calvarium. Granules of calcium phosphate compounds can be packed to fit the calvarial defect [19], but they are difficult to contain as the area requires reconstruction and lacks structural stability. To improve these mechanical properties, we developed a new, malleable, biodegradable osteoconductive material. It consists of tricalcium phosphate particles bound by a gelatin set by glutaraldehyde mediated cross-linking. The gelatin is partially hydrolyzed collagen extracted and purified from porcine skin. The gelatin not only can hold the tricalcium phosphate particles in place, but can also increase the mechanical strength of the implant. We call this novel material 'GTG composite'. The purpose of this study is to investigate the tissue compatibility, biodegradation and osteoconductive activity of this material in rabbit calvarial defects up to three months.

\section{Materials and methods}

\subsection{Implant materials}

Tricalcium phosphate powder (Merck, Germany) was placed in a platinum crucible and sintered at $1000^{\circ} \mathrm{C}$ for $1 \mathrm{~h}$ and then cooled to room temperature. The sintered powder was crushed in a grinding bowl and sieved in 40-60 mesh. Tricalcium phosphate particles with an average grain size of 100-150 $\mu \mathrm{m}$ were obtained for material preparation.

$5 \mathrm{gm}$ gelatin powder (Signa, USA) was dissolved in $25 \mathrm{ml}$ deionized distilled water at $65^{\circ} \mathrm{C}$ by water bath. This homogeneous gelatin solution was mixed with $15 \mathrm{gm}$ tricalcium phosphate particles. 4\% glutaraldehyde solution (Sigma, USA) was added to the tricalcium phosphate/gelatin mixture for gelatin matrix cross-linking. The composite was manually packed into $15 \times 15 \times 1.5 \mathrm{~mm}$ cylindrical Tef lon molds and dried overnight in an oven to make preformed materials. The composite was sterilized by autoclaving prior to use.

\subsection{Experimental procedure}

Thirty-two mature New Zealand white rabbits, weighing $3-3.5 \mathrm{~kg}$, underwent full-thickness excision of the parietal bone, which forms the majority of the cranial vault. The rabbits were anaesthetized with intramuscular injection of sodium pentobarbital, $40 \mathrm{mg} \mathrm{kg}^{-1}$. The head of each rabbit was shaved and disinfected with Betadine. The cranial surface was exposed by a midline incision, and the overlying parietal periosteum was excised. $15 \times 15 \mathrm{~mm}$ circular, full-thickness defect of the parietal bone was created with a drilling burr in a slow-speed dental handpiece supplemented with $0.9 \%$ physiologic saline. The dural and superior sagittal sinus were not violated (Fig. 1).

Twenty rabbits were reconstructed with a composite composed of tricalcium phosphate and glutaraldehyde crosslinked gelatin (GTG composite). The GTG composite was easily molded to the calvarial bone defect and did not require any fixation (Fig. 2). Twelve rabbits were left unreconstructed as negative controls. The periosteum was closed with 5-0 vicryl and the skin was closed with 4-0 nylon. The rabbits were ambulatory within 2-3 h following surgery. Post operative analgesics and antibiotics were not required.

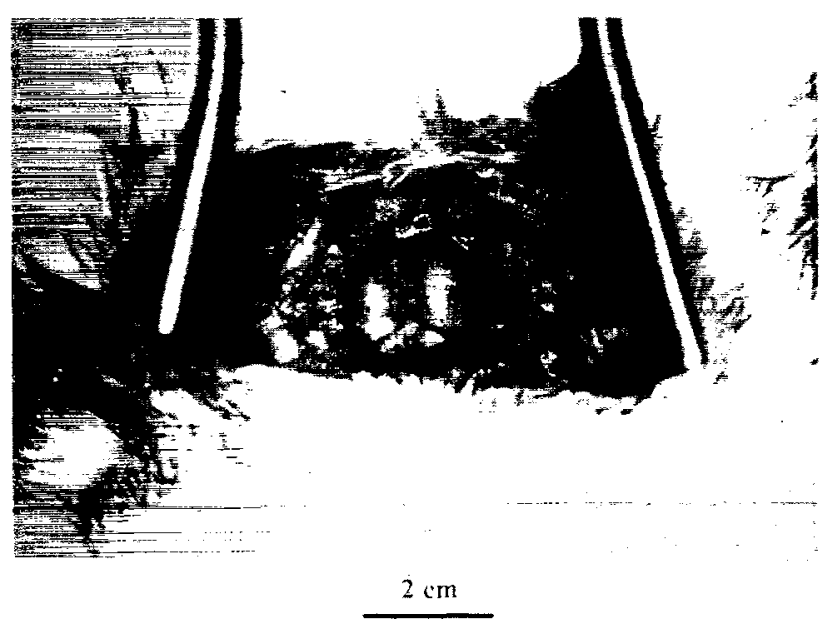

Fig. 1. A $15 \times 15 \mathrm{~mm}$, full-thickness, parietal calvarial bone defect. The dural and superior sagittal sinus were not violated.

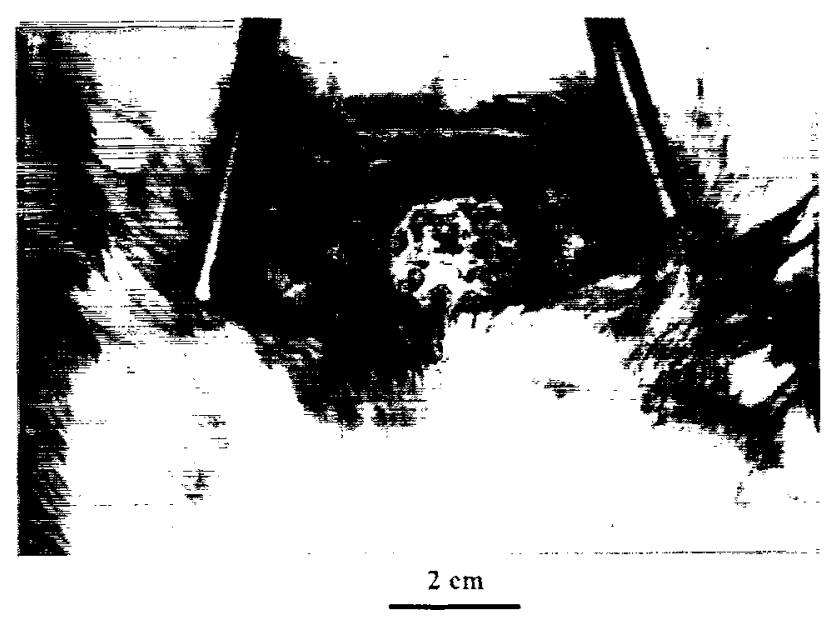

Fig. 2. The composite was easily molded to the defect. No fixation was necessary. 


\subsection{Histological evaluation}

Animals were killed by an overdose of sodium pentobarbital, at 2 weeks, 1, 2 and 3 months after surgery. Five reconstructed and 3 unreconstructed rabbits were examined for each time period.

Craniectomy sites with $2-3 \mathrm{~mm}$ contiguous bone were removed from each skull. Specimens were immediately placed into vials with $70 \%$ ethanol, and prepared for analysis. After $24 \mathrm{~h}$ in $70 \%$ cthanol, specimens were radiographed in a cabinet X-ray machine (Ohmic OM-603, Tokyo) using high contrast X-ray film at $28 \mathrm{KV}, 3 \mathrm{MA}$, for $40 \mathrm{~s}$. The specimens were then decalcified, embedded in paraffin, cut into $5 \mu \mathrm{m}$ coronal sections, and stained with haematoxylin and eosin ( $\mathrm{H}$ and $\mathrm{E}$ ) for transmitted light microscopy study.

\section{Results}

All animals in the experimental and control groups survived the entire duration of the experiment. There were no wound infection, scalp effusion or haematoma, and none of the composites became extruded.

\subsection{Gross examination}

On gross examination of the whole calvarium, the GTG composites were intimately incorporated with the surrounding host bone. The line of demarcation separating the GTG composite from the adjacent host calvarium became less distinct with time. There was also a gradual loss of composite volume (Fig. 3). These changes were directly proportional to the length of time the composite had been in place. The brain underlying the composite did not show evidence of cortical inflammation or scar formation.

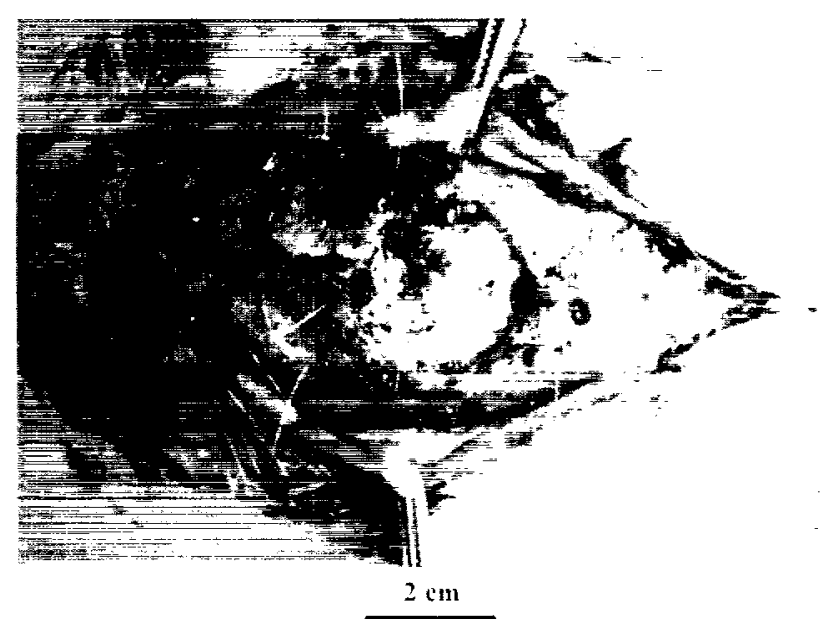

Fig. 3. Three months after implantation of composite. The composite was intimately incorporated with the host bone with a gradual loss of composite volume.

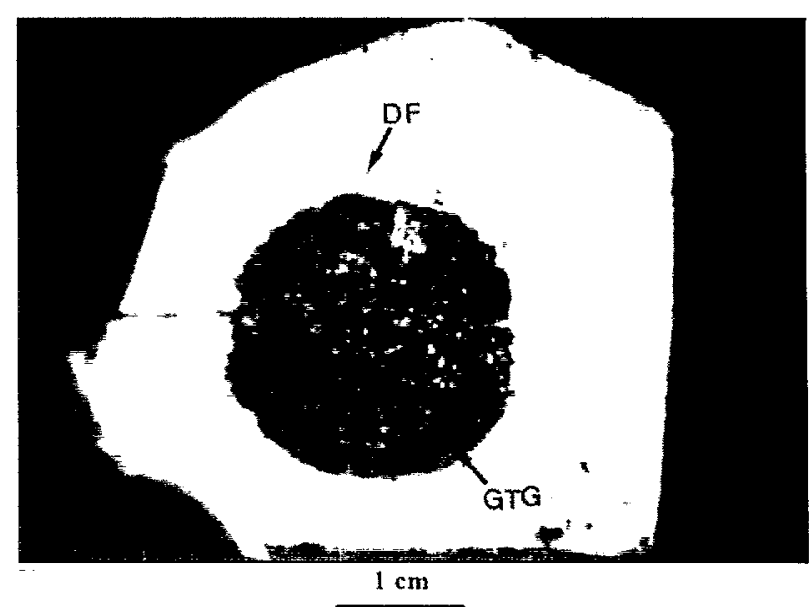

Fig. 4. Radiograph of calvarial defect 2 weeks after implantation of composite. Most of the tricalcium phosphate particles in the composite are clearly visualized. DF, calvarial defect; GTG, composite.

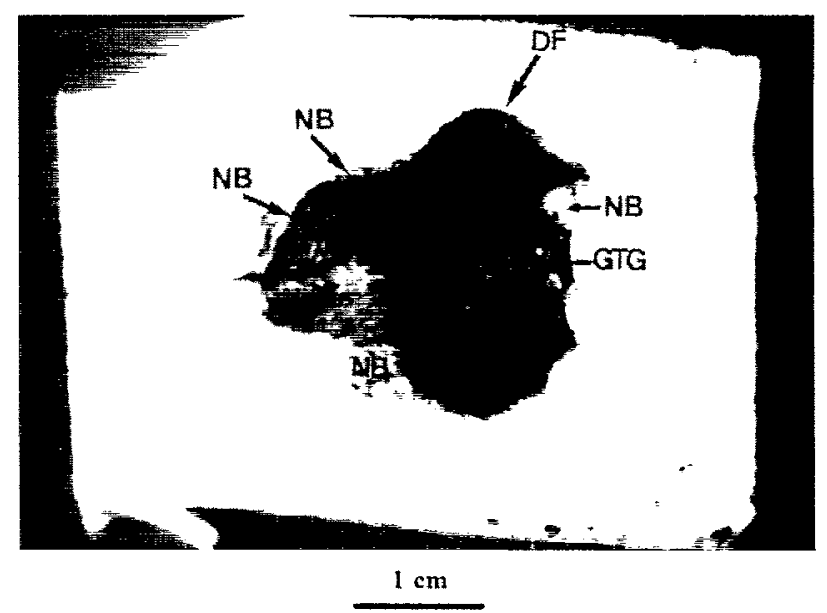

Fig. 5. Radiograph of calvarial defect 3 months after implantation of composite. Most of the tricalcium phosphate particles in the composite are degraded. New bone is laid down in a centripetal direction with a central radiolucent area. DF, calvarial defect; GTG, composite; NB, new hone.

\subsection{Radiographic analysis}

In the serial postoperative radiographs, we found that the GTG composites tend to biodegrade with time. The degradation was coupled with new bone deposition. In the reconstructed groups, there was a progressively increasing amount of radiopaque material in the calvarial defect. The newly formed bone was laid down from the edge of the defect, in a centripetal direction, and obscured the original margin of the calvarial defect. However, none of the reconstructed groups showed complete calcification, all had a central radiolucent area (Figs, 4 and 5). The radiopaque material found in the control groups was only minimal, with clear definition of defect margin.

\subsection{Histological observation}

Examination of the $\mathrm{H}$ and $\mathrm{E}$ stained sections of the craniectomy sites revealed significantly greater amounts of new 


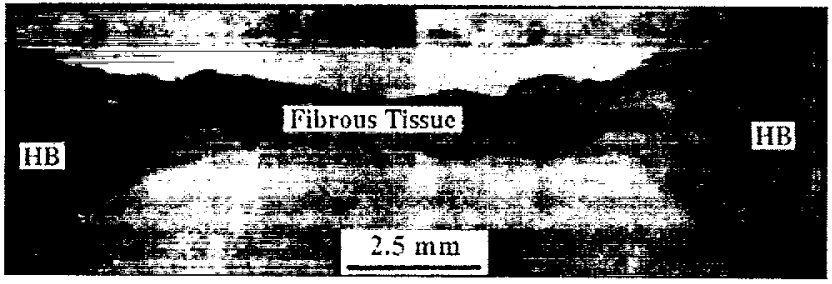

Fig. 6. H and $\mathrm{E}$ stained $(\times 10)$ section of unreconstructed defect, 3 months after cranicetomy. Note only fibrous connective tissue bridged the defect. $\mathrm{HB}$, host bone.

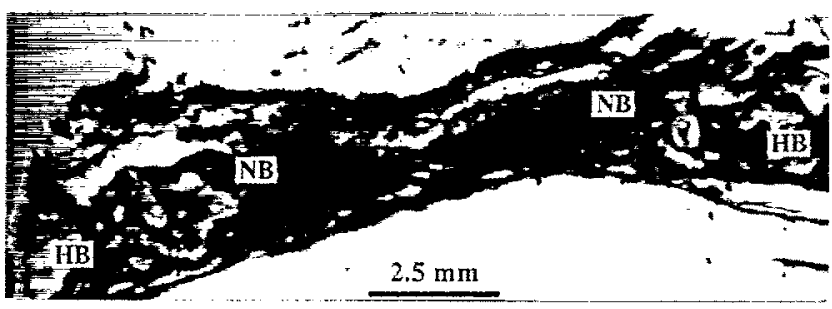

Fig. 7. H and $E$ stained $(\times 10)$ section of reconstructed defect, 3 months after implantation. Note significant amount of composite was replaced by new bone. $\mathrm{HB}$, host bone; $\mathrm{NB}$, new bone.

bone ingrowth in the reconstructed groups than in the controls. Up to three months after surgery, the unreconstructed controls demonstrated only a bridge of fibrous connective tissue across the bone defect with a minimal amount of new bone formation around the edges of the defect (Fig. 6). Histologic evaluation confirmed progressive growth of new bone into the bone defect in the reconstructed groups. At one month after implantation, the process of new bone replacement of the GTG composite began with new bone appearing near the bone-composite interface. This process was more advanced with time, and at three months after implantation, a significant amount of GTG composite was replaced by new bone (Fig. 7). Although there was ingrowth of new bone from the margin, bridging was incomplete even at three months after surgery, and fibrous connective tissue filled the remainder of the defect. Histologic evaluation also confirmed progressive biodegradation of the GTG composite. At one month after implantation, signs of composite degradation appeared at the periphery of the composite. The degradation was coupled with new bone deposition (Fig. 8). At two months after implantation, there was an appearance of more new bone and acellular matrix replacing the composite (Fig. 9). We think that this acellular matrix may come from differentiating osteoblasts. At three months after implantation, most of the composite was replaced by new bone and a bone-like substance that may be formed by calcification of the acellular matrix (Fig. 10). Foreign body or inflammatory cellular response to the GTG composite was minimal in the histologic evaluation.

\section{Discussion}

There is a considerable interest in developing osteoconductive materials to perform the functions of autogenous bone

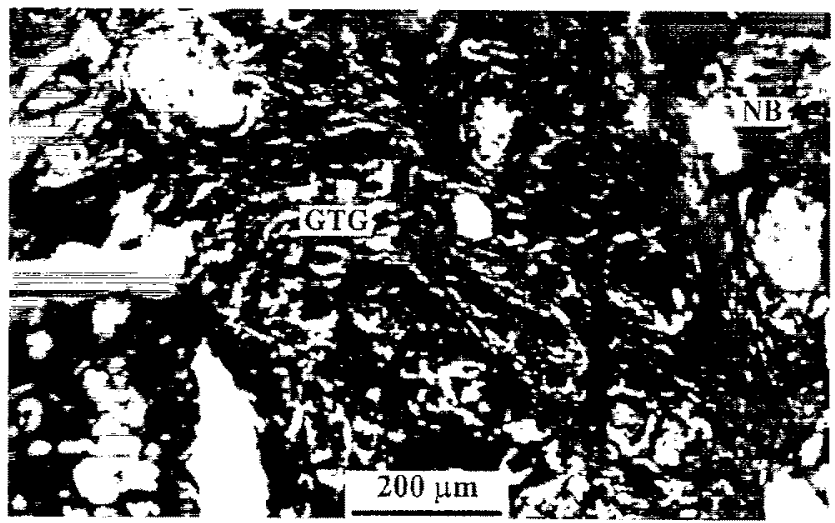

Fig. 8. H and E stained $(\times 40)$ section of composite, one month after implantation. Degradation was coupled with new bone deposition at the periphery. GTG, composite; NB, new bone.

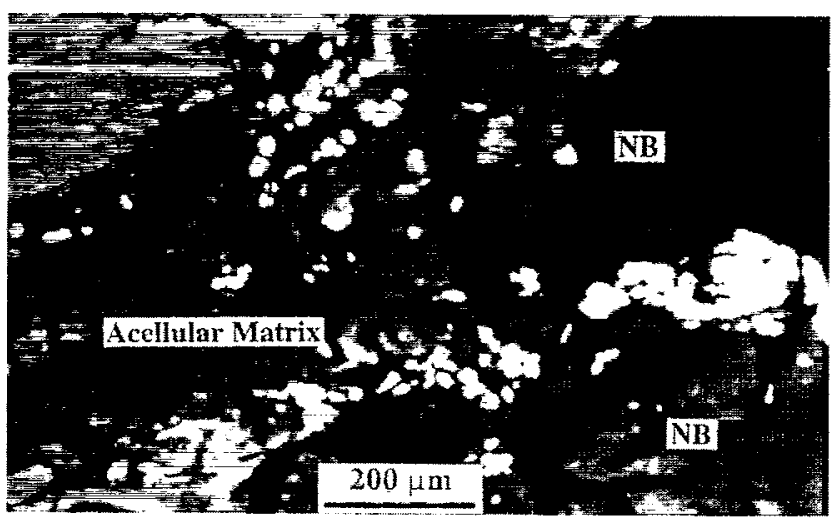

Fig. 9. $\mathrm{H}$ and $\mathrm{E}$ stained $(\times 40)$ section of composite, 2 months after implantation. The composite was replaced by new bone and acellular matrix. NB, new bone.

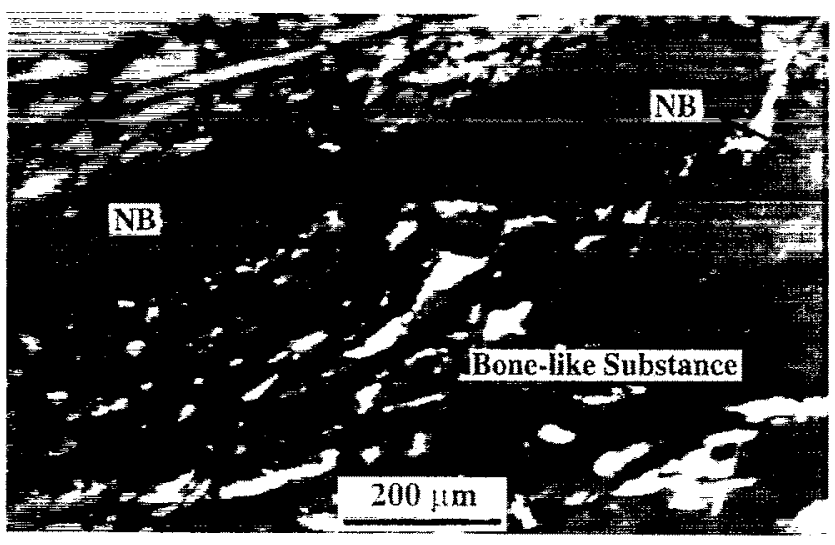

Fig. 10. $\mathrm{H}$ and $\mathrm{E}$ stained $(\times 40)$ section of composite, 3 months after implantation. The composite was replaced by more new bone and bone-like substance, which may be formed by calcification of the acellular matrix. NB, new bone.

graft in calvarium defect reconstruction. Evidence strongly points to the conclusion that materials composed of calcium phosphates are probably the most biocompatible osteoconductive materials known. They are composed mainly of calcium and phosphate ions, the same ions which make up the bulk of natural bone mineral. They can serve as a scaffold for 
bone ingrowth and demonstrate excellent tissue compatibility with the surrounding bone and underlying meninges. The principal limitation of calcium phosphate materials in calvarium defect reconstruction is their mechanical properties. When shaped in porous blocks, they are quite brittle and nonmalleable, can be easily fractured and are difficult to shape so that they accurately replace calvarium defects. Granules of calcium phosphate can be easily packed into a calvarium defect without shaping, but they are difficult to contain within the reconstructed area and lack the structural stability. To prevent migration of granules from the reconstructed site, a number of binding materials such as bovine collagen, fibrin glue and calcium sulphate (plaster of Paris) have been combined with the calcium phosphate materials $[20,21]$. None of these mixtures has proven ideal. Constantino et al. [22] have employed cement of calcium phosphate in cat calvarium defect reconstruction. However, application of this cement was limited by its hydrophilic properties. If it was placed into a field where adequate haemostasis had not been achieved, the cement tended to absorb the ambient fluid and lose its shape.

To improve the mechanical properties of calcium phosphate osteoconductive materials, we developed a new composite. It consists of tricalcium phosphate particles bound by a gelatin set by gultaraldehyde cross-linking. Gelatin, the partially hydrolyzed collagen extracted and purified fromporcine skin, not only can hold the tricalcium phosphate particles in place, but can also increase the mechanical strength of the composite, so that it can be easily molded to the calvarium defect without fracture [23]. We used a $15 \times 15 \mathrm{~mm}$, fullthickness, calvarium defect model in the rabbit to study the biocompatibility, biodegradation and osteoconductive activity of this composite up to three months. A defect of this size in a rabbit will not spontaneously heal during a lifetime and is thus defined as a critical-sized defect [24,25]. The assessment involves serial post operative gross examinations, radiographs and histologic evaluations.

The results of this study, we believe, are significant.

1. The composite is malleable, unlike the ceramic form of calcium phosphate, it can be easily molded into the calvarium defect without fracture.

2. The composite demonstrates good tissue biocompatibility. It can be intimately incorporated with the surrounding bone with time, elicits little inflammatory response, and has no adverse effect on the underlying brain.

3. The composite demonstrates good osteoconductive activity. Radiographic and histologic evaluations confirm progressive growth of new bone into the calvarium defect in the composite reconstructed group.

4. The composite is biodegradable. Histologic evaluation confirms progressive replacement of the composite by new bone, acellular matrix and bone-like material, in a centripetal direction. Osteoconductive material should be biodegradable, thereby permitting gradual remodelling of the ingrowth new bone.
The fate of the released calcium and phosphate from a biodegraded composite has been the subject of study. The results of these studies point to the conclusion that the calcium and phosphate derived from these composites enter the body pool and are utilized in a normal fashion [26-28].

Despite concerns about toxicity of some of the ingredients in this composite, specifically glutaraldehyde $[29,30]$, our unpublished data from co-culturing of this composite with rabbit osteoblasts in vitro revealed that the toxic effect of gutaraldehyde released from the composite can be eliminated by soaking of the composite in distilled water for four days before use.

Although the extent of bone ingrowth in this composite was insufficient to bridge the $15 \times 15 \mathrm{~mm}$ calvarial defect completely, and with fibrous connective tissue filling the remainder of the defect, we believe this composite is valuable in clinical application. The most exciting role of this composite will be as a carrier for bone inductive agents such as demineralized bone matrix or bone morphogenetic protein [31-40]. According to Lucas et al. [41], an osteoinductive agent alone without a carrier, would fail to elicit bone formation. The bone morphogenetic protein, when implanted without a carrier, tends to diffuse too rapidly, before bone induction could occur. It would be advantageous to have a biodegradable, osteoconductive material which may help to maintain the osteoinductive agent at the wound bed, and also acts as an anchorage platform, allowing attachment of the osteocompetent cells from the wound site. We are working to develop materials composed of GTG composite and osteoinductive agents.

In summary, we have tested a new calcium phosphate osteoconductive composite which fulfils most of the criteria for an ideal osteoconductive material. Judicious use of this composite, with full understanding of its limitations, offers the promise of generating new materials for calvarial defect reconstruction in high tension electric burn injury of the head.

\section{Conclusions}

GTG composite has been proven to have a good biocompatibility and could release nutrition elements for the growth of osteoblast in vitro cell culture test. In the study, the calvarial defect model was designed for in vivo evaluation of the GTG composite as bone substitute. From the gross examination of the whole calvarium, the GTG composites were intimately incorporated with the surrounding host bone. The brain underlying the composite did not show the evidence of cortical inflammation or scar formation. The newly formed bone was laid down from the edge of defect, in a centripetal direction, and obscured the original margin of the calvarial defect. However, none of the reconstructed groups showed complete calcification, all had a central radiolucent area. The radiopaque material found in the control groups was only minimal, with clear definition of defect margin. The results of this study indicated that the composite is malleable, unlike 
the ceramic form of calcium phosphate, it can be easily molded into the calvarium defect without fracture. The composite demonstrates good tissue biocompatibility. It can be intimately incorporated with the surrounding bone with time, elicits little inflammatory response, and has no adverse effect on the underlying brain. Radiographic and histologic evaluations confirm progressive growth of new bone into the calvarium defect in the composite reconstructed group. Histologic evaluation confirms progressive replacement of the composite by new generation bone, acellular matrix and bone-like material, in a centripetal direction. Osteoconductive material should be biodegradable, thereby permitting gradual remodelling of the ingrowth new bone.

\section{Acknowledgements}

The authors wish to express gratitude to the National Science Council for their financial support in the study. We would also like to thank Dr Chung Shih, associate professor, Department of Anatomy and Biology, National Defense Medical Center, Taipei, Taiwan, for his assistance in the radiographic evaluation.

\section{References}

[1] B. Siverberg, J.C. Brains, G.D. Verdi, R.D. Acland, Microvascular reconstruction after electrical and deep thermal injury, J. Trauma. 26 (1986) 128-134.

[2] W.B. Nickell, M.J. Jurkiewicz, K.E. Salyer, Repair of skull defects with autogenous bone, Arch. Surg. 105 (1972) $431-433$.

[3] D.J. Prolo, K.P. Burres, W.T. Mclaughlin, et al., Autogenous skull cranioplasty: fresh and preserved (frozen) with consideration of the cellular response, Neurosurgery 4 (1979) 18-29.

[4] S.A. Wolie, Autogenous bune grafts versus alloplasteic material in maxillofacial surgery, Clin. Plast. Surg. 9 (1982) 539-540.

[5] M.S.B. Edwards, D.K. Ousterhout, Autogenic skull bone grafts to reconstruct large or complex skull defects in children and adolescents, Neurosurgery 20 (1987) 273-280.

[6] P.N. Manson, W.A. Crawley, J.E. Hoopes, Frontal cranioplasty: risk factors and choice of cranial vault reconstructive material. Plast. Reconstr. Surg. 77 (1986) 888-900.

[7] K.E. Salyer, J. Barach, C.A. Squier, E.L. Gendler, K.M. Kelly, Cranioplasty in the growing caninc skull using demincralizes perforated bone, Plast. Reconstr. Surg. 96 ( 1995) 770-779.

[8] J.W. Ferro, Experimental evaluation of ceramic calcium phosphate as a substitute for bone grafts, Plast. Reconstr. Surg. 63 (1979) 634640.

[9] M. Jarcho, Calcium phosphate ceramics as hard tissue prosthetics, Clin. Ortho. Relat. Res. 157 (1981) 259-278.

[10] T.J. Flatley, K.L. Lynch, M. Benson, Tissue response to implants of calcium phosphate ceramic in the rabbit spine, Clin. Ortho. Relat. Res. 179 (1983) 246-252.

[11] A. Uchida, S.M.L. Nade, E.R. McCartney, W. Ching, The use of ceramies for bone replacement, J. Bone Joint Surg. 66B (1984) 269_ 275.

[12] J.O. Hollinger, G.C. Battistone, Biodegradable bone repair materials, Clin. Ortho. Relat. Res. 207 (1986) 290-305.

[13] R.W. Bucholz, A. Carlton, R.E. Holmes, Hydroxyapatite and tricalcium phosphate bone graft substitute, Ortho. Clin. North. Am. 18 (1987) 323-334.
[14] R.E. Holmes, H.K. Hagler, Porous hydroxyapatite as a bone graft substitute in cranial reconstruction: a histometric study, Plast. Reconstr. Surg. 81 (1988) 662-671.

[15] G.A. Pettis, L.B. Kaban, J. Glowacki, Tissue response to composite ceramic hydroxyapatite/demineralized bone implants, J. Oral Maxillofac. Surg. 48 (1990) 1068-1074.

[16] H.A. Hoogendoom, W. Renooij, L.M.A. Akkermans, V.J. Visser P. Wittebol, Long-term study of large ceramic implants (porous hydroxyapatite) in dog femora, Clin. Ortho. Relat. Res, 187 (1984) 261-288.

[17] W. Renooij, H.A. Hoogendoom, W.J. Visser, et al., Bioresorption of ceramic strontium-85 labeled calcium phosphate implants in dog femora, Clin. Ortho. Relat. Res. 197 ( 1985 ) 272-285.

[18] J.F. Piecuch, J. Goldberg, C.V. Shastry, R.B. Chrzanowski, Compressive strength of implanted porous replamineform hydroxyapatite, J. Biomed. Mater. Res. 18 (1984) 39-45.

[19] N.G. Georgiade, J. Hanker, G. Ruff, S. Levin, The use of particulate hydroxyapatite and plaster of Paris in aesthetic and reconstructive surgery, Aesth. Plast. Surg. 17 (1993) 85-92.

[20] W.K. Harvey, J.L. Pincock, V.J. Matukas, et al., Evaluation of subcutaneously implanted hydroxyapatite-avitene mixture in rabbits, $\mathrm{J}$. Oral. Maxillofac. Surg. 43 (1993) 277-284.

[21] C.E. Rawlings IM, R.H. Wilkins, J.S. Hanker, et al., Evaluation in eats of new material for cranioplasty: a composite of plaster of Paris and hydroxyapatite, J. Neurosurg. 69 (1988) 269-275.

[22] P.D. Constantino, C.D. Friedman, K. Jones, L,C. Chow, G.A. Sisson, Experimental hydroxyapatite cement cranioplasty, Plast. Reconstr. Surg. 90 (1992) 174-187.

[23] T.N. Gerhart, A.A. Renshaw, R.L. Miller, R.J. Noecker, W.C. Hayes, In vivo histologic and biomechanical characterization of a biodegradable particulate composite bone cement, J. Biomed. Mater. Res. 23 (1989) 1- 16

[24] J.W. Frame, A convenient animal model for testing bone substitute materials, J. Oral. Surg. 38 (1980) 176-180.

[25] J.P. Schmitz, J.O. Hollinger, The critical size defect as an experimental model for craniomandibulofacial nonunions, Clin, Ortho. Relat, Res. 20 (1975) 365-368.

[26] W.A. Mors, E.J. Kaminski, Osteogenic replacement of tricalcium phosphate ceramic implants in the dog palate, Arch. Oral. Biol. 20 (1975) 365-368.

[27] H.U. Cameron, I. Macnab, R.M. Pilliar, Evaluation of a biodegradable ceramic, J. Biomed. Mater. Res. 11 (1977) 179-186.

[28] T. Shima, J.T. Keller, M.M. Alvira, F.H. Mayfield, S.B. Dunsker, Anterior cervical disectomy and interbody fusion: an experimental study using a synthetic tricalcium phosphate, J. Neurosurg. 51 (1979) 533-538.

[29] D.P. Speer, M. Chvapil, C.D. Eskelson, J. Ulreich, Biological effects of residual glutaraldehyde in glutaraldehyde-tanned collagen biomaterials, J. Biomed. Mater. Res. 14 (1980) 753-764.

[30] L.H. Lynn, H. Lee, D.T. Cheung, M.E. Nimni, Biochemical changes and cytotoxicity associated with the degradation of polymeric glutaraldehyde derived crosslinking, J. Biomed. Mater. Res. 24 (1990) $1185-1201$.

[31] D.E. Mark, J.O. IIollinger, C. Ilastings, et al,, Repair of calvarial nonunions by osteogenin, a bone-inductive protein, Plast. Reconstr. Surg. 86 (1990) 623-630.

[32] M.R. Urist, A. Lietze, E. Dawson, Beta-tricalcium phosphate delivery system for bone morphogenetic protein, Clin. Orthop. Relat. Res. 187 (1984) $227-280$.

[33] M.R. Urist, O. Nilsson, J, Rasmussen, et al., Bone regeneration under the influence of a bone morphogenetic protein (RMP) beta tricalcium phosphate (TCP) composite in skull trephine defects in dogs, Clin. Orthop. Relat. Res. 214 (1987) 295-304.

[34] C.J. Damine, J.R. Parson, J.J. Benedict, D.S. Weisman, Investigation of a hydroxyapatite and calcium sulfate composite supplemented with an osteoinductive factor, J. Biomed. Mater. Res. 24 (1990) 639-651.

[35] A.E. Turk, K. Ishida, J.A. Jensen, J.S. Wollman, T.A. Miller, 
Enhanced healing of large cranial defects by an osteoinductive protein in rabbits, Plast. Reconstr. Surg. 92 (1993) 593-600.

[36] R. Kenley, L. Marden, T. Turek, L. Jin, E. Ron, J.O. Hollinger, Osseous regeneration in the rat calvarium using novel delivery systems for recombinant human bone morphogenetic protein-2 (th BMP-2), J. Biomed. Mater. Res. 28 (1994) 1139-1147.

[37] L. Marden, J.O. Hollinger, A. Chaudhri, T. Turek, R.G. Schaub, E. Ron, Recombinant human bone morphogenetic protein- 2 is superior to demineralized bone matrix in repairing craniotomy defects in rats, J. Biomed. Mater. Res. 28 (1994) 1127-1138.

[38] C.J. Damien, J.R. Parson, A.B. Prewett, D.C. Rietveld, M.C. Zimmerman, Investigation of an organic delivery system for demi- neralized bone matrix in a delayed-healing cranial defect model, $\mathrm{J}$. Biomed. Mater. Res. 28 (1994) 553-561.

[39] H.F. Sailer, E. Kolb, Application of purified bone morphogenetic protein (BMP) preparations in cranio-maxillofacial surgery, J. Cranio-Maxillo-Fac. Surg. 22 (1994) 191-199.

[40] E.R. Ashby, G.H. Rudkin, K. Ishida, T.A. Miller, Evaluation of a novel osteogenic factor, bone cell stimulating substance, in a rabbit cranial defect model, Plast. Reconstr. Surg. 98 (1996) 420-426.

[41] P.A. Lucas, G.T. Syftestad, V.M. Goldberg, A.I. Caplan, Ectopic induction of cartilage and bone by water-soluble proteins fron bone using a collagenous delivery vehicle, J. Biomed. Mater. Res. 23 (A1) (1989) 23-29. 